\title{
PROPAGATION OF GUIDED WAVES IN A HOLLOW CIRCULAR CYLINDER APPLICATION TO NON DESTRUCTIVE TESTING
}

\author{
Djili Sonia \\ Characterization and instrumentation laboratory \\ Scientific and Technical Center of Research on Welding and Control \\ BP64 route de Delly Brahim Cheraga, Algiers, Algeria. \\ Tel: 00213361850 \\ Fax: 00213361850 \\ Email: sonia_djili@yahoo.fr \\ Boubenider Fouad \\ Laboratory of physics of materials \\ Team "waves and acoustic" \\ University of sciences and technology Houari Boumedienne B.P. 32 El Allia- Bab Ezzouar - 16111 - Algiers- \\ (Algeria) \\ Tel: 0021321247912 Poste 076 \\ Fax: 0021321247344 \\ Email: fboubenider@yahoo.fr
}

\begin{abstract}
In this paper, we study the propagation of guided waves in a tube and their interaction with surface defects. Axisymmetric (longitudinal and torsional) modes and nonaxisymmetric (flexural) modes are found and represented in phase and group velocity dispersion diagrams. The mode chosen to be generated is the second longitudinal mode $\mathrm{L}(0,2)$, for the frequency-thickness product equal to $1 \mathrm{MHz} . \mathrm{mm}$, at this product, the radial component of displacement is very small with regard to the axial component so, the attenuation is less important. According to our experimental results the $\mathrm{L}(0,2)$ mode is sensitive to the depth variation and to the defect circumference. A relation of proportionality between the amplitude of the signal reflected by the defect and its depth was established. The results of the modal decomposition method, concerning the amplitude of the modes diffracted by circumferential defects according to the circumference of the defect, were presented and compared with the experimental measures results.
\end{abstract}

KEY WORDS: guided waves, longitudinal mode, non destructive testing, tube.

\section{Introduction}

Ultrasonic guided waves inspection has developed rapidly as a tool for materials assessment, structural integrity tests and manufacturing control. Ultrasonic non-destructive testing receives a lot of attention for such advantages as low cost, ease for operation, high sensitivity, etc. Guided waves are made of a superposition of bulk waves such as longitudinal and shear waves [1]. For a waveguide such a pipe, the longitudinal and shear waves are reflected back and forth between boundaries, which lead to guided wave propagation along the waveguide. Guided waves of different mode or frequency have different acoustic field distribution. Guided waves will be reflected if they impinge on a defect. However, defect classification and sizing by guided waves is still a major problem to be resolved due to the complexity of wave propagation characteristics. The dispersive nature of guided waves, the mode conversion phenomenon at the defects, the variation of wave structures of each mode at different frequencies, etc. all contribute to the difficulty of quantitative evaluation of the defects.

Our work consists, in the theoretical part, to plot dispersion curves which will allow us to choose the best mode adapted to the inspection of our structure. In our case, chosen mode is the longitudinal $\mathrm{L}(0,2)$ mode. This mode is very attractive for testing for several reasons: it is practically non-dispersive over a wide bandwidth, that is to say its velocity does not vary significantly with frequency, so that the signal shape and amplitude are retained as it travels; it is the fastest mode so that any unwanted mode converted signals arrive after it has been 
received; and it is equally sensitive to internal or external defects at any circumferential location. In the experimental part, we generate the $\mathrm{L}(0,2)$ mode and we identify all echoes reflected by the edge of tube. The variation of the acoustic pressure according to the circumference and the depth of the defect are studied. Besides, the results obtained by the modal decomposition method, concerning the amplitude of the modes diffracted by circumferential defects according to the circumference of the defect, will be presented and compared with the experimental measures results.

\section{Dispersion curves and mode choice:}

Guided waves in hollow cylinders were first completely studied by Gazis [2]. Guided waves in a hollow cylinder have a double infinite number of modes and the energy is constrained inside the tube wall. Therefore, guided waves in a hollow cylinder are very complicated to study. The modes are labelled $\mathrm{L}(0, n), \mathrm{T}(0, \mathrm{n})$ and $\mathrm{F}(\mathrm{m}, \mathrm{n})$ and they, respectively, refer to longitudinal, torsional and flexural modes. The first index ' $\mathrm{m}$ ' indicates the order of harmonic variation of displacement and stresses around the circumference and the second index ' $\mathrm{n}$ ' is a counter variable. Axisymmetric modes like L $(0,1)$ and $\mathrm{L}(0,2)$ bear similar characteristics with Lamb waves. For guided waves in a hollow cylinder, higher order modes L $(0,3), \mathrm{L}(0$, 4 ), etc. will appear. Each higher mode has a cut-off frequency. The evolution of wave structures of different modes at different frequencies has been discussed by GAZIS [2]. When the frequency goes very high, $\mathrm{L}(0,1)$ and $\mathrm{L}(0,2)$ modes will degenerate into surface waves in a pipe. Surface waves are a superposition of $\mathrm{L}(0,1)$ and $\mathrm{L}(0,2)$ at very high frequency. The material parameters of tube, used for plot of the dispersion curves is listed in Table.1

\begin{tabular}{|c|c|c|c|}
\hline$\rho\left(\mathrm{g} / \mathrm{cm}^{3}\right)$ & $\mathrm{V}_{\mathrm{L}}(\mathrm{m} / \mathrm{s})$ & $\mathrm{V}_{\mathrm{T}}(\mathrm{m} / \mathrm{s})$ & $v$ \\
\hline 8,9 & 4628,5 & 2223,5 & 0,35 \\
\hline
\end{tabular}

Table. 1: Material parameters used for of the pipe

In Fig. 1, one method for representing the dispersion relation of an isotropic copper tube with a radius mid plane $\mathrm{R}=10,5 \mathrm{~mm}$ and a wall thickness $\mathrm{h}=1 \mathrm{~mm}$ is depicted.

Normalized frequencies are plotted as a function of normalized wave numbers.

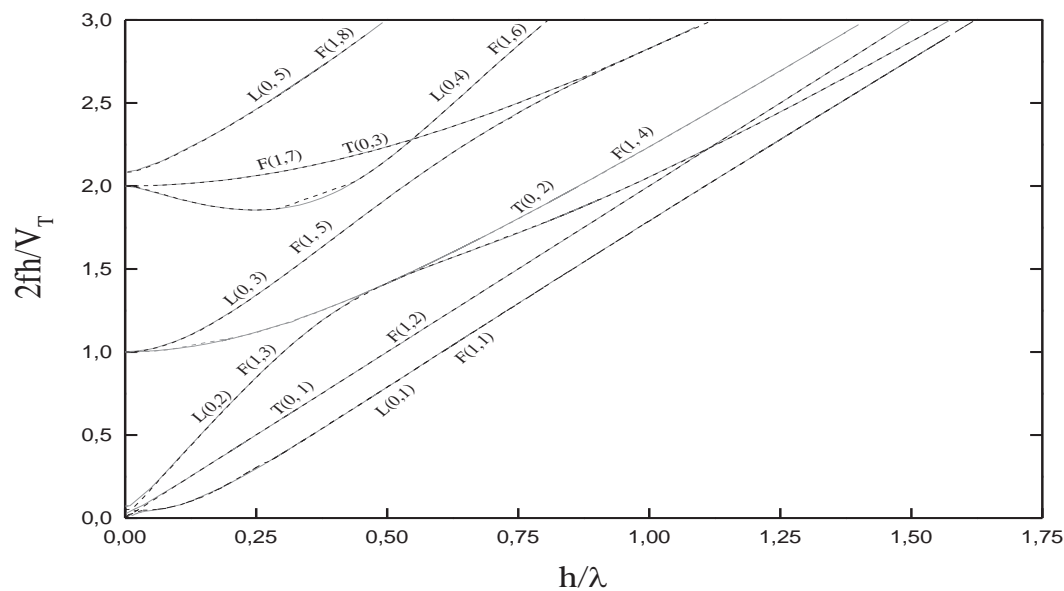

Fig. 1: frequency spectrum for a copper tube the axisymmetric longitudinal and torsional wave modes for $n=0$ (dashed black curves) and the flexural modes for $\mathrm{n}=1$ (solid gray lines) are plotted.

$\mathrm{V}_{\mathrm{T}}$ is transversal velocity; $\mathrm{f}$ is the frequency and $\lambda$ the wavelength. 
Another method to display the various modes is shown in Fig. 2 where the normalized phase velocities of the modes are given as a function of normalized frequency.

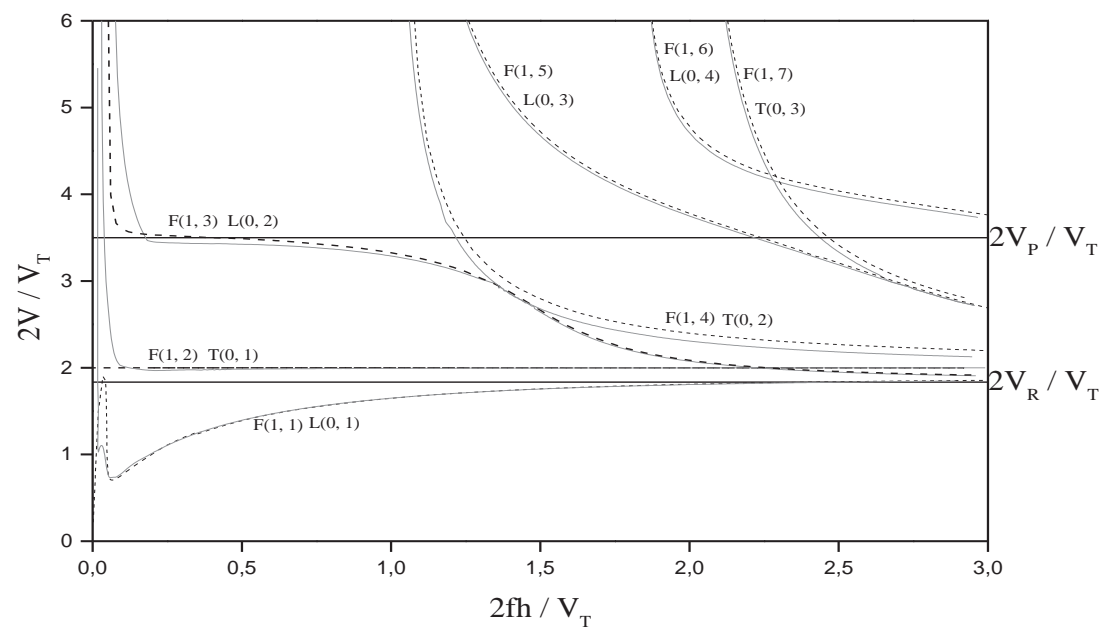

Fig. 2: Phase velocity dispersion curves for a copper tube. The axisymmetric modes (longitudinal and torsional) are plotted as dashed black lines while the gray curves correspond to the flexural modes with $\mathrm{n}=1$.

For a successful guided wave testing, it is necessary to draw the dispersion curves. Any point on dispersion curves can be used for ultrasonic testing, but any mode of guided wave is more sensitive to a special defect type [3]. Some modes are sensitive to coating or surface defects and some others are sensitive to internal defects, and so on. Displacement curves can explain the sensitivity of guided modes to defect types [4]. Axisymmetric modes are in general preferable because they are easier to excite and have relatively simple acoustic fields. Initial practical testing was done using the longitudinal $\mathrm{L}(0,2)$ mode [5][6].

\section{Method of Guided Wave Generation}

Incident angle and frequency are two parameters which can be varied for excitation a desired guided wave testing. Snell's law can be used for calculating the incident angle of the media between the transducer and tube for producing a special guided mode [7], here the $\mathrm{L}(0,2)$ mode, with a defined phase velocity as is followed:

$\theta=\sin ^{-1}\left(\mathrm{~V}_{\mathrm{C}} / \mathrm{V}\right)$

Where $\mathrm{V}_{\mathrm{C}}$ is the velocity of longitudinal ultrasonic wave in the separating media (water), $\mathrm{V}$ is the phase velocity, at the frequency-thickness product chosen, of guided wave mode and $\theta$ is the incident angle in the separating media.

To generate $\mathrm{L}(0,2)$ mode alone, we must orientate the transducer under an incidence angle of $\theta=\sin ^{-1}(1500 / 3779)=23,4^{\circ}$. With $V_{C}=1500 \mathrm{~m} / \mathrm{s}$ is the velocity of longitudinal ultrasonic wave in the water and $V=3779 \mathrm{~m} / \mathrm{s}$ is the phase velocity of the $\mathrm{L}(0,2)$ mode at the frequencythickness product equal to $1 \mathrm{MHz} . \mathrm{mm}$.

The advantage of the immersion method is the possibility to vary the orientation of the $\theta$ angle around $23,4^{\circ}$, until having a signal of maximum amplitude. 

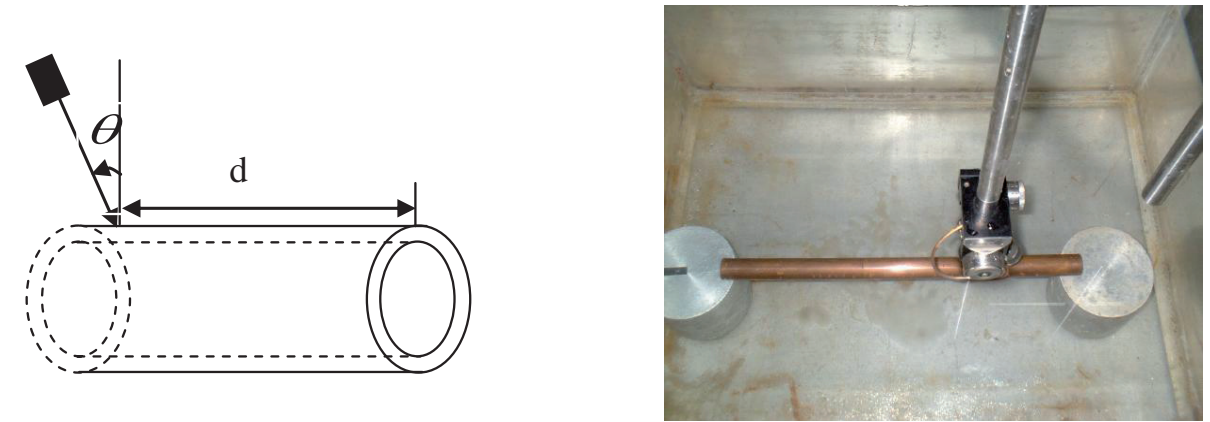

Fig. 3: $\theta$ is the incidence angle and $d$ is the distance between the guide side and the impact of the acoustic beam issued

\section{Experimental Results}

For the experimental trials a cooper tube, having $1 \mathrm{~mm}$ thickness and average radius equal to $10,5 \mathrm{~mm}$.

1/ Propagation of the $\mathrm{L}(0,2)$ mode on a sound tube:

The reflection of the $\mathrm{L}(0,2)$ mode on the side of sound tube gives here under

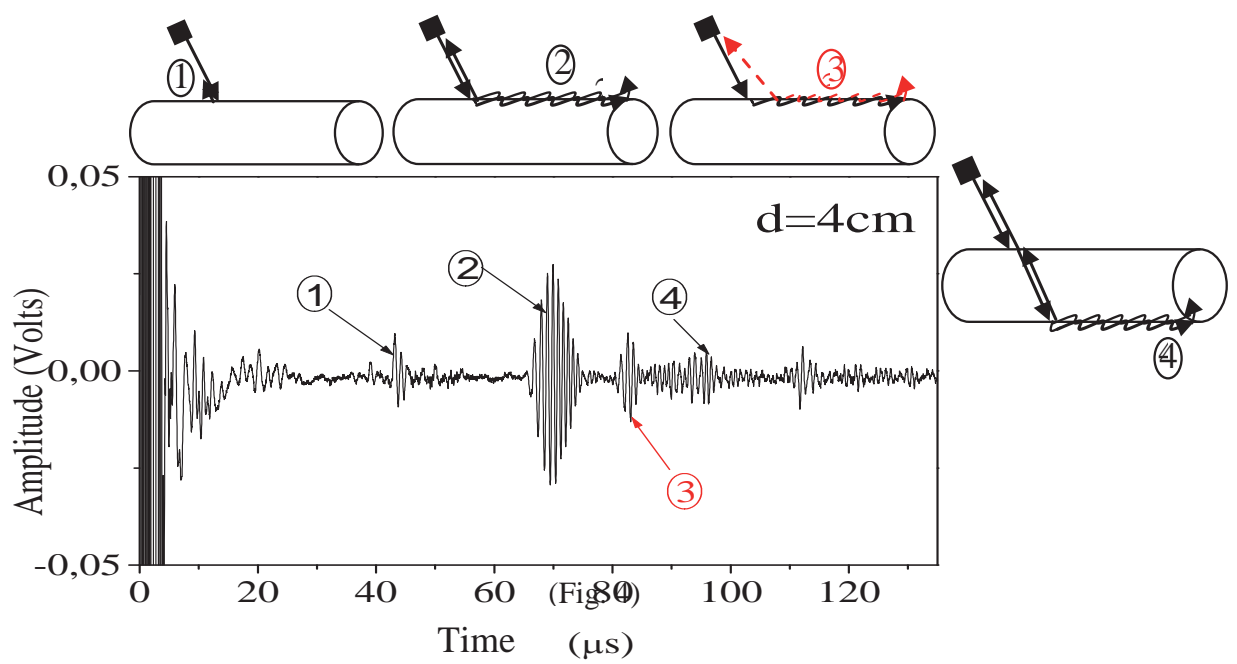

Fig. 4: Identification of the different echoes coming back of the side.

The first echo represents the wave retro diffusion by the surface of the tube. The second is the reflection of $L(0,2)$ mode by the edge, the distance covered by the wave is the same go and back way. The third echo, was identified as, the first mode of compression $\mathrm{L}(0,1)$, reflected by the edge of the tube; it's the $\mathrm{L}(0,2)$ mode conversion, the wave in that case has a different itinerary on the way back, it returns on the transducer under an angle of $48^{\circ}$.

The echo number four, represents the reflection of $\mathrm{L}(0,2)$ mode on the edge after its crossing through the tube. 
Signals below represent the reflection of the $\mathrm{L}(0,2)$ mode on the edge, for various distances impact edge.
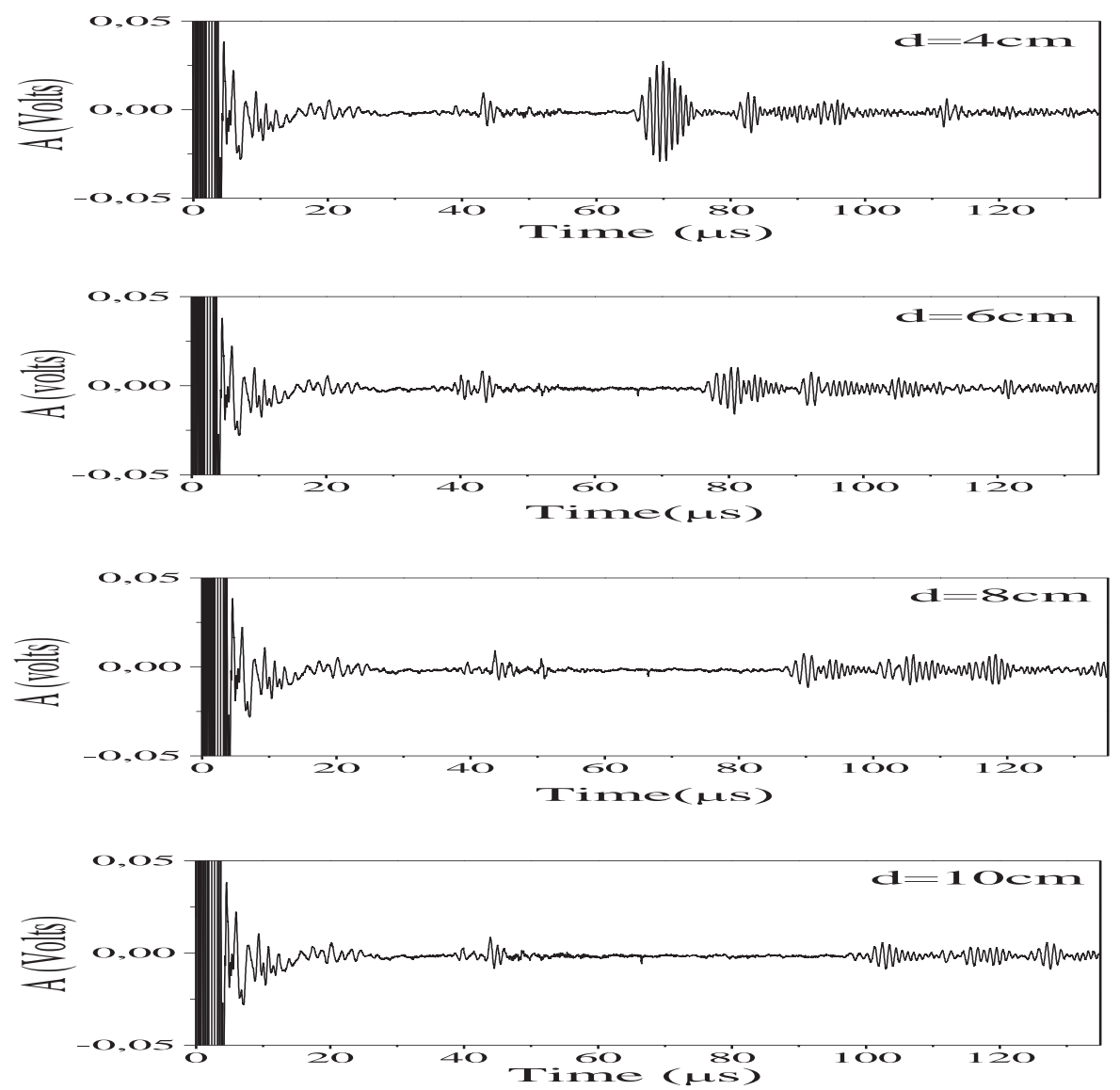

Fig. 5: signals coming from the reflection, on the guide side, for different distances d.

In fact, the mode, in this band of frequency, is not of pure compression mode, so, we expect a progressive attenuation. 


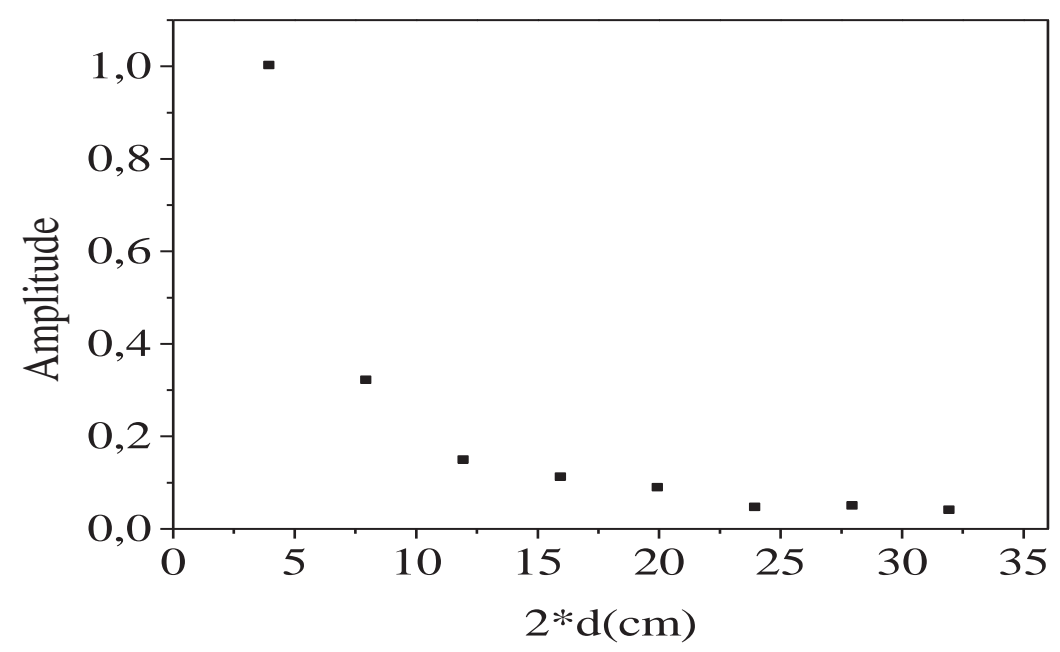

Fig. 6: variation of the amplitude of the reflective signal by the side according to the $\mathrm{d}$ distance.

The amplitude varies exponentially, it is a result which we expected [8].In fact, this phenomena is due to the wave diffusion in the liquid.

$2 / \mathrm{L}(0,2)$ mode propagation on tube with cracks:

Tube was performed with notch defects, with notch defects, with < defect circumference through wall depth as reference defects.

The reflection of guided waves is governed by very different rules from those of volume waves. For guided waves, it is possible to detect defects with very small size, in comparison with the wave length.

At the frequency thickness product f.h $=1 \mathrm{MHz} \cdot \mathrm{mm}$, the $\mathrm{L}(0,2)$ mode has a wavelength of $\lambda=3,8 \mathrm{~mm}$.
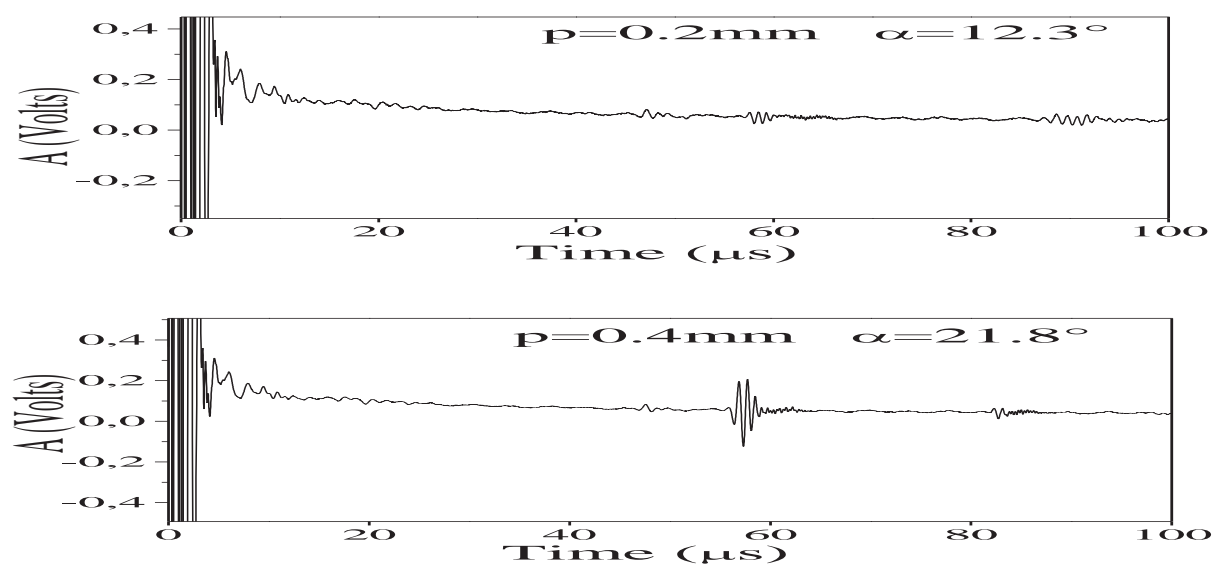

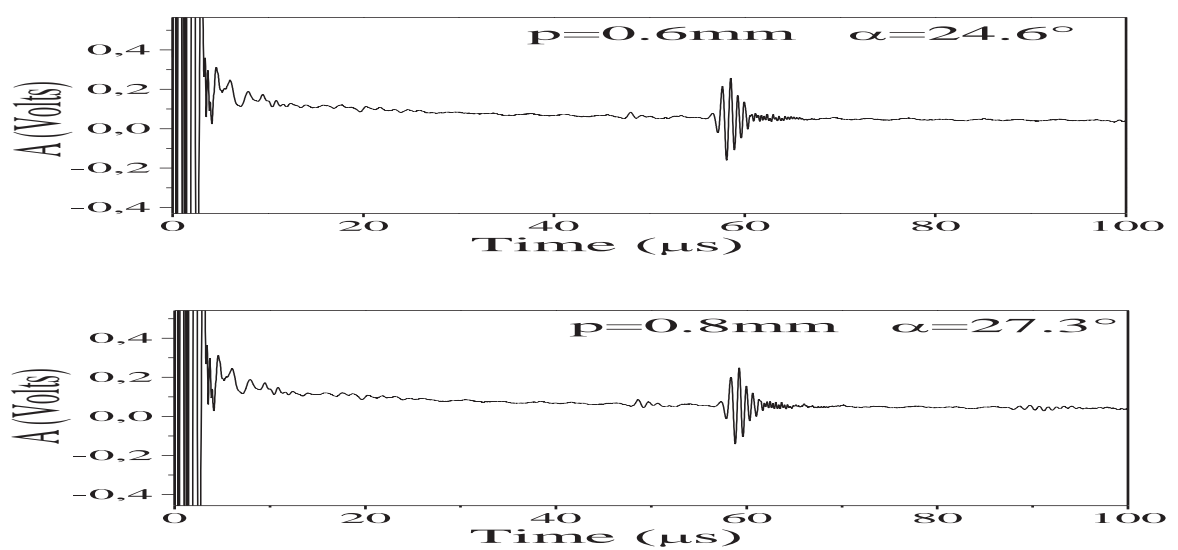

Fig.7: Reflection of the $\mathrm{L}(0,2)$ mode, on crack of different size.

The precedents statements, confirm what we advance previously the $\mathrm{L}(0,2)$ mode is able to detect defects of very low dimensions, in front of the wavelength, a defect of depth $\mathrm{p}=0.2 \mathrm{~mm}$ and of circumference $\alpha=12.3^{\circ}$ has could be detected.

A study of the signal amplitude variation, reflected by the defect, according to the circumference of this last will be exposed, the sample is a tube with only one defect, of depth $\mathrm{p}=0,5 \mathrm{~mm}$ and variable circumference $\alpha$.

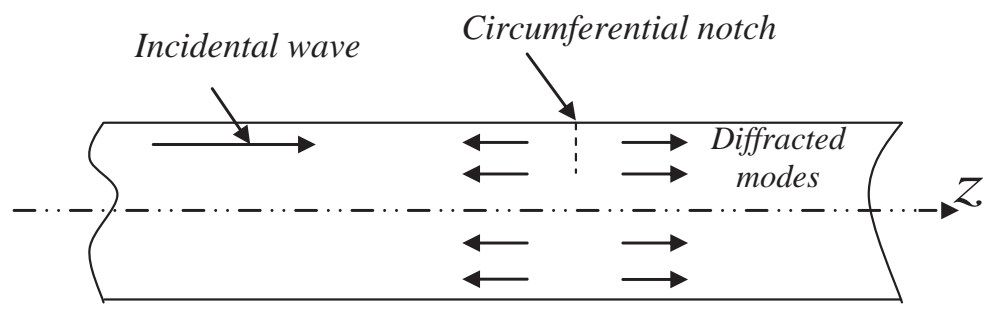

Fig.8: diffraction of the $\mathrm{L}(0,2)$ mode by a notch circumferential

The method of the parameters $\mathrm{S}$ makes it possible to connect the amplitude $b_{v \lambda}^{N}$ of the guided modes diffracted by an in homogeneousness on the tube to the amplitudes $a_{\mu k}^{M}$ has incidental modes, by the relation:

$b_{v g}^{N}(\lambda=g)$ : amplitudes of the guided modes diffracted towards the left.

$b_{v d}^{N}(\lambda=d)$ : amplitudes of the guided modes diffracted towards the line.

$a_{\mu g}^{M}(k=g)$ : amplitudes of the incidental modes towards the left.

$a_{\mu d}^{M}(k=d)$ : amplitudes of the incidental modes towards the line.

$\mathrm{N}$ and $v$ : being respectively the circumferential order and the number of mode of the incidental modes.

$\mathrm{M}$ and $\mu$ : being respectively the circumferential order and the number of mode of the incidental modes. 
In the present study only one mode noted $(\bar{M}, \bar{\mu})$ of amplitude a has is incidental by the left, on defect, several modes $(N, v)$ are considered with noted respective amplitudes $b_{v \lambda}^{N}$.

The sum is thus reduced in the only one term connecting the amplitude of a wave diffracted to that of the incidental mode.

That is to say:

$$
b_{v \lambda}^{N}=S_{v \lambda, \bar{\mu} k}^{N, \bar{M}} a_{\bar{\mu} k}^{\bar{M}}
$$

Or the parameter $\mathrm{S}$ is the amplitude ratio between a diffracted wave $(N, v)$ and the incidental wave $(\bar{M}, \bar{\mu})$.

In the case or the incidental mode are the $\mathrm{L}(0,2)$ mode and the diffracted modes is the longitudinal modes, the relation which gives the amplitude of the various modes diffracted by the defect is the following one:

$$
S_{\bar{v} \lambda, \bar{\mu} k}^{\bar{N}, 0}=\operatorname{cste} \times \Phi(\bar{N}, \alpha)
$$

with

$$
\Phi(\bar{N}, \alpha)=\frac{\alpha \cos (\bar{N} \alpha)}{\bar{N}^{2}}-\frac{\sin (\bar{N} \alpha)}{\bar{N}^{3}}
$$

For an incidental $\mathrm{L}(0,2)$ mode, the author of the article [9] used the modal decomposition to trace the variation of the amplitudes of the longitudinal modes diffracted by a circumferential crack, according to the size of this one.

In this work, the curve of variation, amplitude of the $\mathrm{L}(0,2)$ mode diffracted by notches, various circumferences, is compared with our experimental results. We used, several tubes on which, of the notches of various circumferences $\alpha$ and of constant depth $\mathrm{p}=0,5 \mathrm{~mm}$, were carried out.

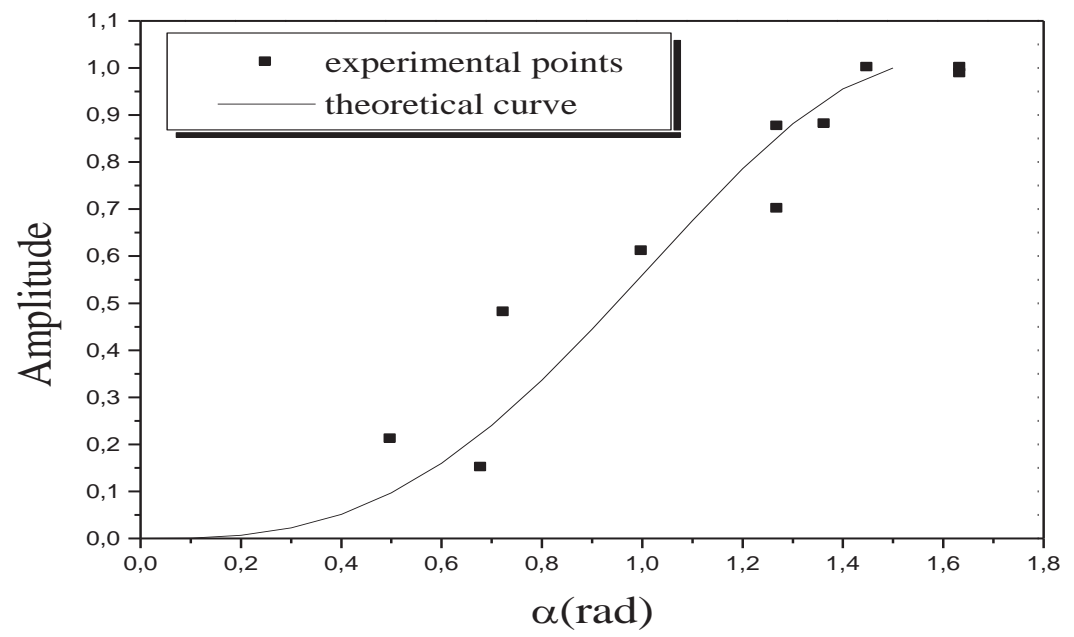

Fig. 9: Relation between the amplitude of the reflexive signal and size circumferentially of the defect, experimental results $\mathbf{m}$ ), predictions of the modal decomposition method ( ). 
The Fig.9 watches a good agreement between the theoretical predictions of the modal decomposition and the experimental results.

We studied, the behaviour of the amplitude of the longitudinal mode $\mathrm{L}(0,2)$, with respect to the depth; for a circumference of $\alpha=\pi$ fixed, in experiments. Our results are summarized on the Fig. 10 figure.

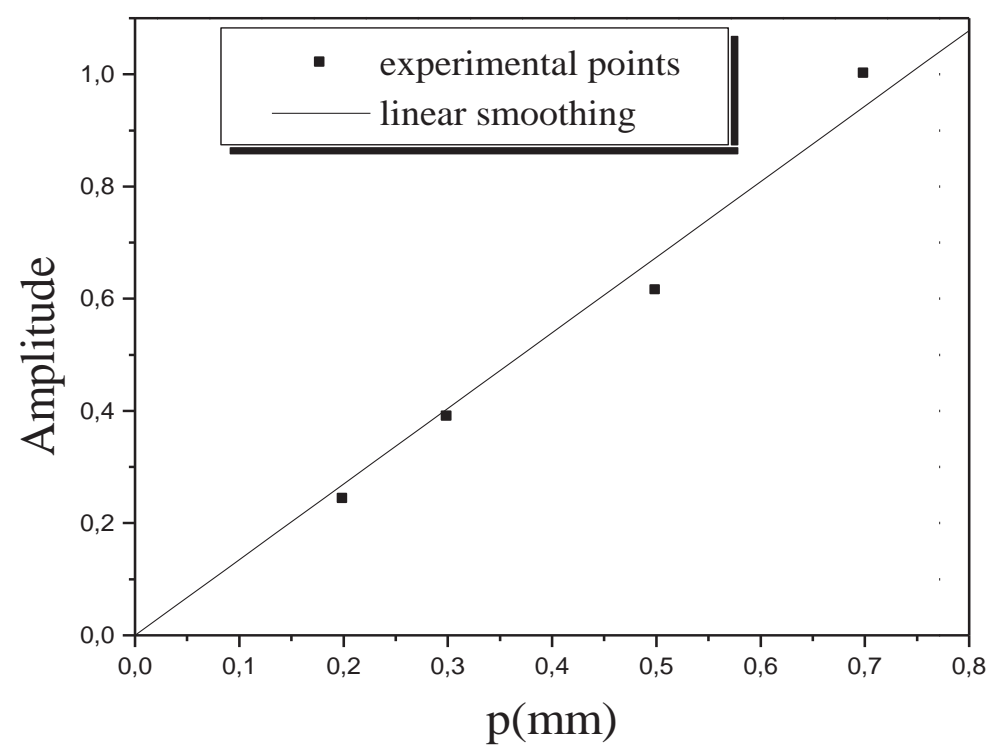

Fig.10: Relation between the amplitude of the reflexive signal and the depth of the defect experimental results. A relation of proportionality, linearity even, between the depth of the defect and the amplitude of the signal reflected by this last is observed. This result confirms what was found, theoretically and in experiments in the case of circumferential waves [10] [11] and in the case of longitudinal waves L $(0,2)[12]$.

\section{Conclusions}

After having studied, the various families likely to be propagated in a tube, and having plotted the curves of dispersion their speeds of phase and group, one could, to choose the mode to be generated and the experimental method to use.

To generate the mode of compression $\mathrm{L}(0,2)$, one used a piezoelectric transducer of centre frequency $1 \mathrm{MHz}$. We choose a tube of which the thickness is of $1 \mathrm{~mm}$. The point of operation chosen is on the slightly dispersive zone of the curve of dispersion of the second longitudinal mode.

The experimental part was used to us to include/understand, the behaviour of the L $(0,2)$ mode on a submerged tube (for a product frequency-thickness of $1 \mathrm{MHz} . \mathrm{mm}$ ) at the time of interaction with circumferential defects. Identification of the various echoes coming from the edge, and those reflected by a circumferential defect was done. It was noted, that one could detect defects, of very low size, in front of the wavelength. It, moreover, was noted a sensitivity of the emitted mode, with the variation depth and the circumference of the defect.

\section{REFERENCES}

1. Rose, J.L (1995) Recent advances in guided wave NDE. IEEE Ultrasonics Symposium, 761-770. 
2. Gazis, D.C. (1959) Three dimensional investigation of the propagation of waves in hollow circular cylinders. J.Acoust.Soc.Am. 31, 568-578.

3. Rose, J.L. (2003) Dispersion curves in guided wave testing. Materials Evaluation. 61, 2022.

4. Pan, E. Rogers, J. Datta, S.K. and Shah, A.H. (1999) Mode selection of guided waves for ultrasonic inspection of gas pipelines with thick coating. Mechanics of Materials. 31, 165174.

5. Demma, A. Cawley, P. Lowe, M. and Roosenbrand, A.G. (2003) The reflection of the fundamental torsional mode from cracks and notches in pipes. J.Acoust.Soc.Am. 114, 611625.

6. Demma, A. Cawley, P. Lowe, M. Roosenbrand, A.G. and Pavlakovic, B. (2004) The reflection of guided waves from notches in pipes: a guide for interpreting corrosion measurements.NDT\&E International. 37, 167-180.

7. Edalati, K. Kermani, A. Nader, B. and Panahi, B. (2005) Defects Evaluation in Lamb Wave Testing of Thin Plates. Middle East Nondestructive Testing Conference \& Exhibition, Manama (Bahrain).

8. Royer, D. and Dieulesaint, E. (1996) Ondes élastiques dans les solides. Tome 1, Paris :Masson.

9. Ditri, J. (1994) Utilization of guided elastic waves for the characterization of circumferential cracks in hollow cylinders. J.Acoust.Soc.Am. 96, 3755-3769.

10. Shan Lin. Koichiro Kawashina. Tosh ih iro Ito. and Hisashi Nagam izo. (2000) Wave propagation analysis by finite element method for flaw sizing of circular pipes. Proc. 15th World Conference on Nondestructive Testing, Roma (Italy).

11. Christine Valle. Marc Niethammer. Jianmin Qu. and Laurence J. Jacobs. (2001) Crack characterization using guided circumferential waves. J.Acoust.Soc.Am. 110, 1282-1290. 12. Lowe, M.J.S. Alleyne, D.N. and Cawley, P. (1998) The mode conversion of a guided wave by a part-circumferential notch in pipe. J.Applied Mechanics. 65, 649-656. 